\title{
The faint supernova remnant G 34.7-0.4 (W44)
}

\author{
F. Mavromatakis ${ }^{1}$, P. Boumis ${ }^{2}$, and C. D. Goudis ${ }^{2,3}$ \\ 1 University of Crete, Physics Department, PO Box 2208, 71003 Heraklion, Crete, Greece \\ 2 Institute of Astronomy \& Astrophysics, National Observatory of Athens, I. Metaxa and V. Pavlou, P. Penteli, 15236 Athens, \\ Greece \\ 3 Astronomical Laboratory, Department of Physics, University of Patras, 26500 Rio-Patras, Greece
}

Received 11 March 2003 / Accepted 18 April 2003

\begin{abstract}
Flux calibrated images of the known supernova remnant G 34.7-0.4 in basic optical emission lines are presented. The low ionization images show a relatively flat flux distribution. The diffuse and patchy morphology of the detected optical emission may indicate the presence of turbulent magnetic fields. Typical observed $\mathrm{H} \alpha+[\mathrm{N}$ II] fluxes are $\sim 8 \times$ $10^{-17} \mathrm{erg} \mathrm{s}^{-1} \mathrm{~cm}^{-2} \operatorname{arcsec}^{-2}$, while the [S II] fluxes are lower around $4 \times 10^{-17} \mathrm{erg} \mathrm{s}^{-1} \mathrm{~cm}^{-2} \operatorname{arcsec}^{-2}$. Emission in the medium ionization line of [O III] $5007 \AA$ is not detected within our sensitivity limits, probably due to the heavy extinction towards the remnant. The long-slit spectra reveal strong [S II] and [N II] emission relative to $\mathrm{H} \alpha$ and moderate [O I] $6300 \AA$ A emission. Shock velocities in the range of $110-150 \mathrm{~km} \mathrm{~s}^{-1}$ and low electron densities are estimated. Archival MSX infrared data show emission in the south and west areas of the remnant matching rather well the optical and radio emission.
\end{abstract}

Key words. ISM: general - ISM: supernova remnants - ISM: individual objects: G 34.7-0.4

\section{Introduction}

The radio emission from G34.7-0.4 (W44) is non-thermal $\left(\alpha \sim-0.4 ; I \sim v^{\alpha}\right)$ and displays a shell like morphology. The shell possesses an ellipsoidal shape with the major axis oriented in the south-east to north-west direction and an extent of $\sim 35^{\prime} \times 26^{\prime}$ (e.g. Jones et al. 1993; Giacani et al. 1997). The strongest filamentary radio emission is observed in the eastern half of the remnant, while Kundu \& Velusamy (1972) found that the projected magnetic field distribution is quite uniform in the northeast areas of the remnant. The X-ray images of the object reveal a centrally brightened morphology with the $\mathrm{X}-$ ray emission bounded by the radio emission (Rho et al. 1994). Temperature measurements of the hot gas lie in the range of $0.7-1.0 \mathrm{keV}$, while the neutral hydrogen column density is $\sim 10^{22} \mathrm{~cm}^{-2}$ (Harrus et al. 1997; Jones et al. 1993). The $21 \mathrm{~cm}$ line observations of Koo \& Heiles (1991) revealed the presence of an H I shell associated with W44 expanding with a velocity of $\sim 150 \mathrm{~km} \mathrm{~s}^{-1}$, and carrying $\sim 8 \times 10^{44} \mathrm{erg}$ of kinetic energy.

Narrow band optical images of the remnant have been presented by Rho et al. (1994) and Giacani et al. (1997) in the emission lines of $\mathrm{H} \alpha+[\mathrm{N} \mathrm{II}]$ and [S II]. These images show the diffuse nature of the optical emission, its low surface brightness and a satisfactory degree of correlation with the radio emission. Interest on this remnant was further raised by the discovery of a $267 \mathrm{~ms}$ pulsar at a distance of $\sim 3 \mathrm{kpc}$ and a characteristic spin-down age of $20000 \mathrm{yr}$ (Wolszczan et al. 1991). Subsequent deep X-ray observations by ASCA and Chandra in

Send offprint requests to: F. Mavromatakis,

e-mail: fotis@physics.uoc.gr the vicinity of the pulsar lead to the discovery of an extended nebulosity. Its spectrum is hard and can be described by a steep power-law model (Harrus et al. 1996; Petre et al. 2002). The emission around the pulsar is considered to originate from a synchrotron nebula powered by PSR B1853+01.

Our current view of W44 consists of a remnant in the radiative phase of its evolution with a radius of $\sim 12 \mathrm{pc}$ at a distance of $\sim 2.5 \mathrm{kpc}$ (Cox et al. 1999). Thermal conduction has, most likely, altered the interior density and temperature distributions from those expected by an adiabatic remnant. The progenitor star exploded $\sim 20000$ yr ago releasing $\sim 10^{51}$ erg into an interstellar medium (ISM) of relatively high density $\left(\sim 3-6 \mathrm{~cm}^{-3}\right)$. The ISM density is not uniform but displays a significant gradient perpendicular to the major axis of the remnant with a scale height of $\sim 22 \mathrm{pc}$ (Cox et al. 1999; Shelton et al. 1999).

In this work we present flux calibrated images in the optical emission lines of $\mathrm{H} \alpha+[\mathrm{NII}]$, [S III] and [O III] as well as long-slit spectra at three different locations of W44. In Sect. 2 we provide information about the observations and data analysis, while in Sects. 3 and 4 the results of the imaging and spectroscopic observations are presented. Finally, we discuss the properties of the supernova remnant and the environment into which lies in Sect. 5.

\section{Observations}

\subsection{Optical images}

The supernova remnant W44 was observed with the $0.3 \mathrm{~m}$ Schmidt-Cassegrain telescope at Skinakas Observatory, Crete, 
Table 1. Spectral log.

\begin{tabular}{lll}
\hline \hline Slit centers & & Exp. times $^{a}$ \\
\hline$\alpha$ & $\delta$ & No of spectra $^{b}$ ) \\
\hline $18^{\mathrm{h}} 55^{\mathrm{m}} 25^{\mathrm{s}}$ & $01^{\circ} 32^{\prime} 04^{\prime \prime}$ & $7200(2)$ \\
$18^{\mathrm{h}} 55^{\mathrm{m}} 59^{\mathrm{s}}$ & $01^{\circ} 05^{\prime} 18^{\prime \prime}$ & $7800(2)$ \\
$18^{\mathrm{h}} 55^{\mathrm{m}} 24^{\mathrm{s}}$ & $01^{\circ} 24^{\prime} 44^{\prime \prime}$ & $7800(2)$ \\
\hline
\end{tabular}

${ }^{a}$ Total exposure times in $\mathrm{s}$.

${ }^{b}$ Number of spectra obtained.

Greece on July 7 and August 7 and 8, 2002. The observations were performed with a $1024 \times 1024$ Thomson CCD which provides a $70^{\prime} \times 70^{\prime}$ field of view and an image scale of $4^{\prime \prime}$ per pixel. The remnant was observed through the $\mathrm{H} \alpha+[\mathrm{N} \mathrm{II}]$, [S II], and [O III] filters. Astrometric solutions were calculated for all data frames using the HST Guide star catalogue (Lasker et al. 1999) and were subsequently projected to a common origin on the sky before any arithmetic operation. The equatorial coordinates quoted in this work refer to epoch 2000.

Standard IRAF and MIDAS routines were employed for the reduction of the data. All frames were bias subtracted and flat-field corrected using a series of well exposed twilight flat-fields. The absolute flux calibration was performed through observations of a series of spectrophotometric standard stars (HR 5501, HR 7596, HR 7950, HR 9087 and HR 8634; Hamuy et al. 1992, 1994). The digital data were retrieved from the European Southern Observatory (ESO) and were folded with the appropriate filter transmission curve, which is known for the specific focal ratio of the $0.3 \mathrm{~m}$ telescope. For the given airmass, exposure time of each calibration star and the instrumental setup, it is possible to predict the expected count rate. A least squares fit for the expected and observed count rates allows us to calculate the zero point magnitude and the atmospheric extinction in the specific filter. These coefficients are then applied to the data frames for absolute flux calibration (see also Jacoby et al. 1987).

\subsection{Optical spectra}

Long-slit spectra were obtained on July 6, 7, and 8, 2002 with the $1.3 \mathrm{~m}$ Ritchey-Cretien telescope at Skinakas Observatory. The data were taken with a 1300 line $\mathrm{mm}^{-1}$ grating and a $800 \times 2000$ SITe CCD covering the range of $4750 \AA-6815 \AA$. The projected slit width on the sky is 7.7 , while its length is $7 ! 9$. The coordinates of the slit centers, the number of available spectra from each location and the total exposure times are given in Table 1. The spectrophotometric standard stars HR 5501, HR 7596, HR 7950, HR 9087, and HR 8634 were observed for the absolute calibration of the source spectra. The spectra were reduced with the IRAF software and specifically, with the packages twodspec, longslit and apextract. The sufficient coverage in airmass of the calibration stars allowed to determine both the sensitivity function of the instrument response and the dependence of the airmass on the wavelength. Subsequently, these corrections were applied to the data spectra.

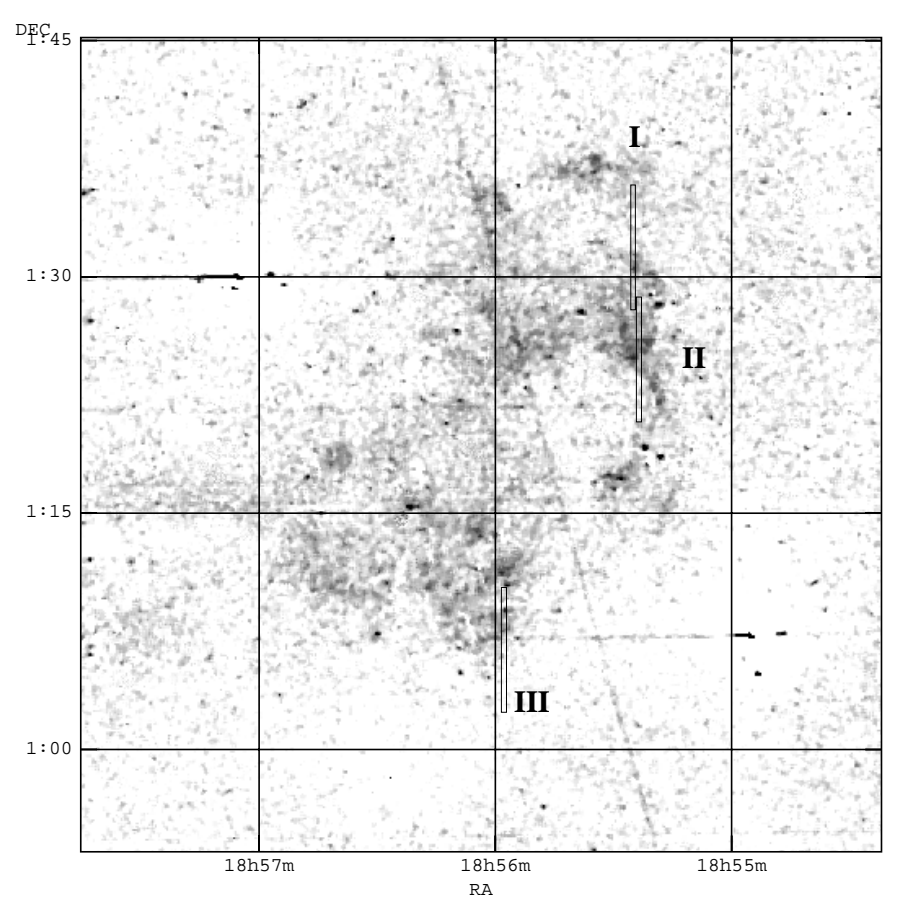

Fig. 1. The diffuse and patchy nature of the emission from $\mathrm{G}$ 34.7-0.4 is the major characteristic of the $\mathrm{H} \alpha+[\mathrm{N} \mathrm{II}]$ image. The shadings run linearly from 0 to $20 \times 10^{-17} \mathrm{erg} \mathrm{s}^{-1} \mathrm{~cm}^{-2} \operatorname{arcsec}^{-2}$ and the long rectangles mark the approximate projection of the slits on the sky. The image has been smoothed to suppress the residuals from the imperfect continuum subtraction. The line crossing the field in, roughly, the south-north direction is due to a satellite pass. The line segments seen near over-exposed stars in this figure and the next figures are due to the blooming effect.

\section{The imaging observations}

\subsection{The $\mathrm{H} \alpha+[\mathrm{NII}]$ and [SII] line images}

The low ionization images covering the $\mathrm{H} \alpha$, [N II], and [S II] lines (Figs. 1 and 2) display a diffuse and patchy morphology already noted by Giacani et al. (1997) and Rho et al. (1994). The flux in each of the $\mathrm{H} \alpha+[\mathrm{NII}]$ and [S II] images appears to have a smooth distribution over the extent of the remnant. Since our images are flux calibrated, we can measure an average $\mathrm{H} \alpha+[\mathrm{N}$ II] flux of $\sim 8 \times 10^{-17} \mathrm{erg} \mathrm{s}^{-1} \mathrm{~cm}^{-2} \operatorname{arcsec}^{-2}$ over several locations of the remnant with faint diffuse emission emitting at a level of $3 \times 10^{-17} \mathrm{erg} \mathrm{s}^{-1} \mathrm{~cm}^{-2} \operatorname{arcsec}^{-2}$. The estimated $\mathrm{H} \alpha$ flux is $\sim 4 \times 10^{-17} \mathrm{erg} \mathrm{s}^{-1} \mathrm{~cm}^{-2} \operatorname{arcsec}^{-2}$ since the [N II] 6548, $6584 \AA$ lines contribute equally well in the $\mathrm{H} \alpha+[\mathrm{N}$ II $]$ filter and we have assumed that the $\mathrm{H} \alpha$ and [N II] fluxes are of comparable strength (e.g. Fesen et al. 1980; Mavromatakis et al. 2002; see also Sect. 4). Measuring the observed sulfur flux over the same locations we find an average value of $4 \times$ $10^{-17} \mathrm{erg} \mathrm{s}^{-1} \mathrm{~cm}^{-2} \operatorname{arcsec}^{-2}$, while the faintest diffuse emission amounts to a flux of $\sim 2 \times 10^{-17} \mathrm{erg} \mathrm{s}^{-1} \mathrm{~cm}^{-2} \operatorname{arcsec}^{-2}$. However, the two sulfur lines do not contribute equally in the filter bandpass and in order to account for this effect we assume a [S II] $6716 \AA$ //[S II] $6731 \AA$ ratio of 1.4, appropriate for middle aged remnants. Consequently, the average total sulfur flux is estimated around $6 \times 10^{-17} \mathrm{erg} \mathrm{s}^{-1} \mathrm{~cm}^{-2} \operatorname{arcsec}^{-2}$. Although 
Table 2. Relative line fluxes.

\begin{tabular}{lllllll}
\hline \hline & Area I & & Area II & \multicolumn{3}{c}{ Area III } \\
\hline Line $(\AA)$ & $F^{a, b}$ & $I_{\text {corr }}^{c}$ & $F^{a, b}$ & $I_{\text {corr }}^{c}$ & $F^{a, b}$ & $I_{\text {corr }}^{c}$ \\
\hline $4861 \mathrm{H} \beta$ & - & 100 & - & 100 & $12(3)$ & 100 \\
$4959[\mathrm{O} \mathrm{III}]_{1}$ & - & - & - & - & - & - \\
$5007[\mathrm{O} \mathrm{III}]_{2}$ & $10^{\mathrm{d}}(3)$ & 901 & - & - & - & - \\
$6300[\mathrm{OI}]_{1}$ & $23(6)$ & 108 & $26(5)$ & 123 & $59(7)$ & 279 \\
$6364[\mathrm{O} \mathrm{I}]_{2}$ & $7(4)$ & 25 & $8(2)$ & 36 & $28(4)$ & 119 \\
$6548[\mathrm{~N} \mathrm{II}]_{1}$ & $28(5)$ & 85 & $26(8)$ & 80 & $20(4)$ & 61 \\
$6563 \mathrm{H} \alpha$ & $100(18)$ & 300 & $100(30)$ & 300 & $100(19)$ & 300 \\
$6584[\mathrm{~N} \mathrm{II}]_{2}$ & $86(15)$ & 249 & $84(25)$ & 242 & $81(15)$ & 234 \\
$6716[\mathrm{~S} \mathrm{II}]_{1}$ & $80(15)$ & 186 & $97(29)$ & 228 & $75(16)$ & 176 \\
$6731[\mathrm{~S} \mathrm{II}]_{2}$ & $59(12)$ & 134 & $71(22)$ & 162 & $50(10)$ & 114 \\
\hline \hline $\mathrm{Absolute} \mathrm{H} \alpha$ flux & 3.8 & & 4.1 & & 2.9 & \\
\hline$[\mathrm{S} \mathrm{II}] / \mathrm{H} \alpha$ & $1.39[0.11]$ & 1.07 & $1.68[0.07]$ & 1.29 & $1.25[0.10]$ & 0.97 \\
{$[\mathrm{~N} \mathrm{II}] / \mathrm{H} \alpha$} & $1.15[0.09]$ & 1.11 & $1.12[0.06]$ & 1.08 & $1.08[0.09]$ & 1.04 \\
{$[\mathrm{~S} \mathrm{II}]_{1} /[\mathrm{S} \mathrm{II}]_{2}$} & $1.36[0.15]$ & 1.38 & $1.36[0.08]$ & 1.40 & $1.51[0.18]$ & 1.55 \\
$n_{\mathrm{e}}(1 \sigma)$ & $<220 \mathrm{~cm}^{-3}$ & & $<140 \mathrm{~cm}^{-3}$ & & $<200 \mathrm{~cm}^{-3}$ & \\
\hline
\end{tabular}

${ }^{a}$ Fluxes uncorrected for interstellar extinction and relative to $F(\mathrm{H} \alpha)=100$.

${ }^{b}$ Listed fluxes are a signal to noise weighted average of the individual fluxes.

${ }^{c}$ Corrected for interstellar extinction assuming $E(B-V)=3.28$ or $c=4.94$.

${ }^{d}$ Detected only in one of the two spectra.

${ }^{e}$ In units of $10^{-17} \mathrm{erg} \mathrm{s}^{-1} \mathrm{~cm}^{-2} \operatorname{arcsec}^{-2}$.

Numbers in parentheses represent the signal to noise ratio of the quoted fluxes.

$1 \sigma$ errors are given in square brackets.

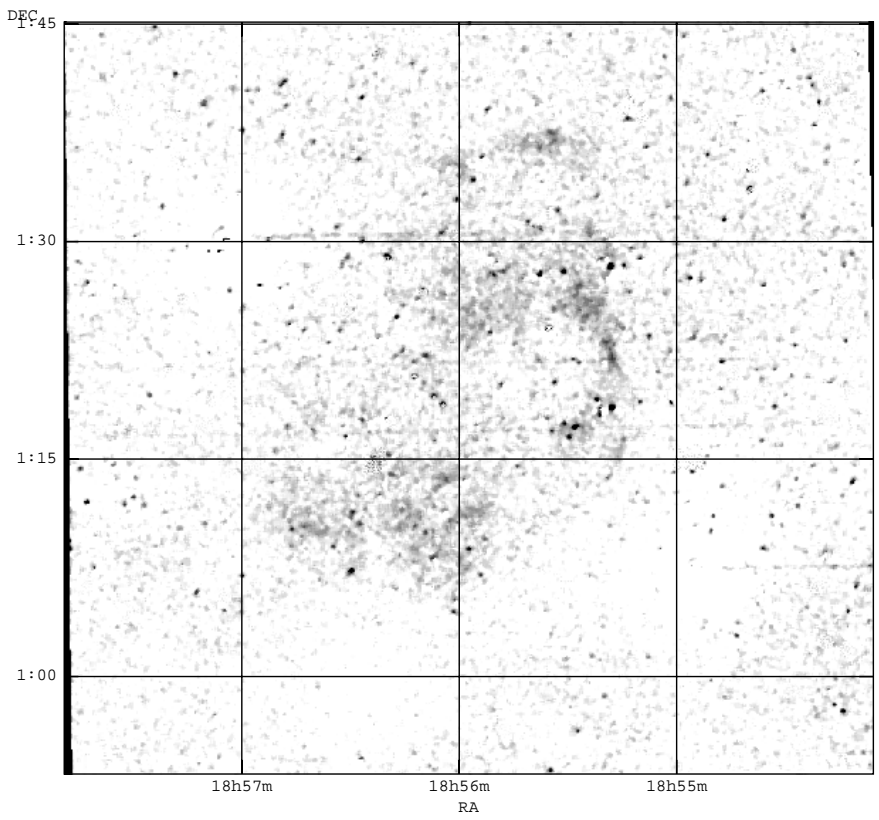

Fig. 2. The field of G 34.7-0.4 imaged with a filter that isolates the sulfur lines at 6716 and $6731 \AA$. The image has been smoothed to suppress the residuals from the imperfect continuum subtraction, while the shadings run linearly from 0 to $10 \times 10^{-17} \mathrm{erg} \mathrm{s}^{-1} \mathrm{~cm}^{-2} \operatorname{arcsec}^{-2}$.

the observed emission in the $\mathrm{H} \alpha+[\mathrm{N} \mathrm{II}]$ and [S II] lines is weak in terms of absolute flux, the sulfur flux is quite strong relative to $\mathrm{H} \alpha$. Using the calibrated images to estimate the observed $[\mathrm{S} \mathrm{II}] / \mathrm{H} \alpha$ ratio, it is found that it ranges from $0.9-1.8$. These values are in agreement with those measured in the available long-slit spectra (Sect. 4).

\subsection{The $[\mathrm{O} I I 1]$ image}

The final [O III] image does not show any emission from the remnant and thus, it is not shown here. The total exposure time of $7200 \mathrm{~s}$ and the hardware configuration used did not allow the detection of possible [O III] $5007 \AA$ emission from W44 and the $3 \sigma$ upper limit set is $1.5 \times 10^{-17} \mathrm{erg} \mathrm{s}^{-1} \mathrm{~cm}^{-2} \operatorname{arcsec}^{-2}$ over its whole area. This upper limit is consistent with the spectroscopic measurements.

\section{The long-slit spectra from $G$ 34.7-0.4}

Long-slit spectra were obtained for the first time from this faint remnant. Approximate slit positions are shown in Fig. 1 where it is seen that locations I, II, and III mainly focus on the brighter areas of the remnant in the south, and north-west. Especially, location II was chosen due to the stronger [S II] emission in that area. The measured fluxes from individual lines along with some derived quantities are given in Table 2 . Since a reliable Balmer decrement measurement towards G 34.7-0.4 is not available, we adopt the statistical relation

$N_{\mathrm{H}}=5.4( \pm 0.1) \times 10^{21} E(B-V) \mathrm{cm}^{-2}$,

given by Predehl and Schmitt (1995) to determine the color excess. Assuming a hydrogen column density of $1.77 \times 10^{22} \mathrm{~cm}^{-2}$ (Tsunemi \& Enoguchi 2002) we find an $E(B-V)$ of 3.28. This column density is consistent with the total galactic column density of $1.7-1.9 \times 10^{22} \mathrm{~cm}^{-2}$, calculated with the FTOOLS command "nh" based on data from Dickey \& Lockmann (1990). The equivalent logarithmic extinction is 4.94 and this value was used to correct all observed spectra for interstellar extinction. 
This, of course, assumes a constant extinction over the remnant but it is possible that the extinction may vary. We note here the presence of LDN 617 in the south areas of G 34.7-0.4 (Lynds 1962). However, in the absence of detailed and accurate measurements we assume it is constant. It is evident from Table 2 that the heavy extinction greatly affects the bluer lines making them very hard to detect.

All spectra are characterized by the low but fairly constant $\mathrm{H} \alpha$ flux and the strong sulfur flux relative to $\mathrm{H} \alpha\left(\left[\mathrm{S} \mathrm{II}_{\mathrm{II}}\right] / \mathrm{H} \alpha \simeq\right.$ 1.1-1.7). The ratio of the two sulfur lines approaches the low density limit in all cases and is estimated that the electron densities over the specific locations are less than $\sim 200 \mathrm{~cm}^{-3}$. Emission at $\mathrm{H} \beta 4861 \AA$ is marginally present in one set of spectra (location III) and may be a spurious detection given its low signal to noise. The failure to detect the $\mathrm{H} \beta$ line prevents us from checking on whether the spectra originate from fully developed or incomplete shock structures. In the following, we will assume that the radiative shock structures are complete. In addition, the medium ionization line of [O III] $5007 \AA$ appears to be absent from the optical spectra. Emission at the $3 \sigma$ confidence level in this line is detected only in one of the two spectra taken from position I suggesting a probable statistical fluctuation (Table 2). The $3 \sigma$ upper limit on the [O III] emission in all spectra is $\sim 0.4 \times 10^{-17} \mathrm{erg} \mathrm{s}^{-1} \mathrm{~cm}^{-2} \operatorname{arcsec}^{-2}$, roughly a factor of 10 less than the typical H $\alpha$ flux. The [O II] 6300, $6364 \AA$ line fluxes from Area III should be treated with caution since there are indications for improper background subtraction.

Note here that the signal to noise ratios given in Table 2 do not incorporate errors due to the calibration process which are $\sim 10 \%$.

\section{Discussion}

The known supernova remnant G 34.7-0.4 lies in the galactic plane at a distance of $2.5 \mathrm{kpc}$ (Cox et al. 1999). As a result, short wavelengths suffer significant extinction rendering optical measurements a difficult task. Earlier optical observations mainly focused on the morphology of the optical emission and provided estimates of the fluxes in the $\mathrm{H} \alpha+[\mathrm{N} \mathrm{II}]$ and $[\mathrm{S}$ II $]$ lines (Giacani et al. 1997).

In this work we present flux calibrated images of this faint remnant in major emission lines and long-slit spectra. The typical flux levels in the $\mathrm{H} \alpha+[\mathrm{N} \mathrm{II}]$ and [S II] images are $\sim 8$ and $4 \times$ $10^{-17} \mathrm{erg} \mathrm{s}^{-1} \mathrm{~cm}^{-2} \operatorname{arcsec}^{-2}$, while emission at the bluer line of [O III] $5007 \AA$ was not detected at the sensitivity limit of our observations. Note that for a color excess of 3.28 (see Sect. 4), the observed intensity of the [O III] line would be 30 times lower than that of the $\mathrm{H} \alpha$ line, even if they had equal intrinsic intensities. It is also evident from the typical fluxes of the low ionization lines that the observed [S II] flux is quite strong relative to $\mathrm{H} \alpha$ (see also Sect. 3.1). The morphology of the optical emission although diffuse and patchy (Figs. 1 and 2) is very well correlated with the radio emission, mainly in the west (Giacani et al. 1997). The nature of the optical emission may be attributed to the presence of radiative shocks seen face-on due to the orientation of the interstellar "clouds" or to a patchy interstellar medium towards G 34.7-0.4. However, line-of-sight extinction variations cannot account for the overall appearance

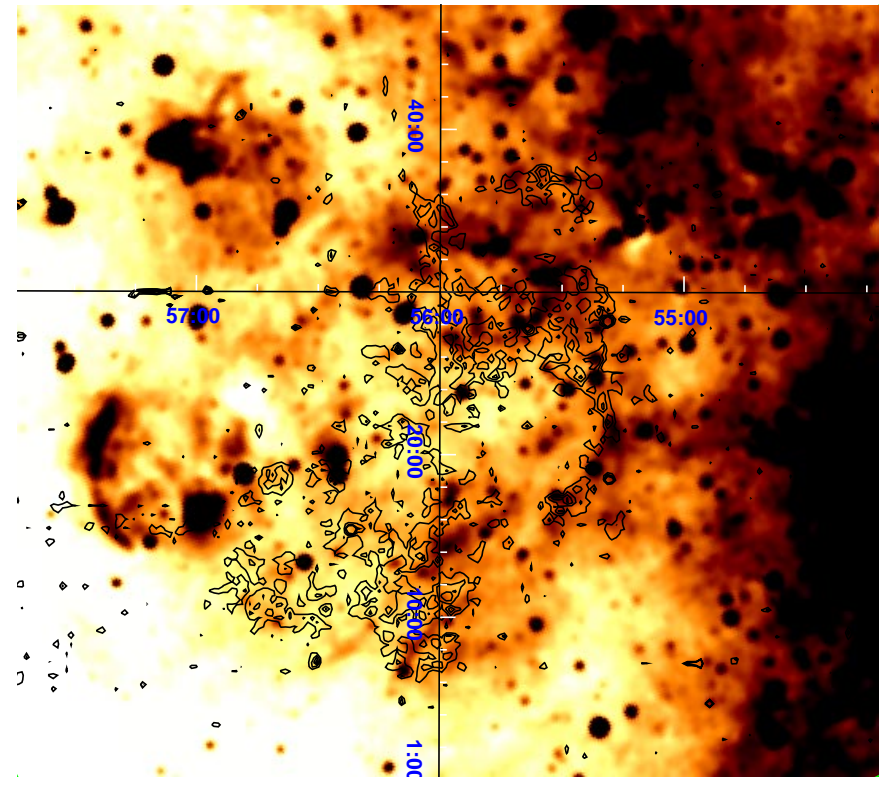

Fig. 3. Dust emission at $8.28 \mu \mathrm{m}$ is shown as a greyscale image, while the contours show the $\mathrm{H} \alpha+[\mathrm{N}$ II $]$ emission. The shadings run linearly from 5.64 to $11.75 \times 10^{-14} \mathrm{erg} \mathrm{s}^{-1} \mathrm{~cm}^{-2} \operatorname{arcsec}^{-2}$ or equivalently from 2.4 to $5.0 \times 10^{-6} \mathrm{~W} \mathrm{~m}^{-2} \mathrm{sr}^{-1}$. Infrared emission detected in the western areas of the remnant seems correlated with the optical emission.

of the remnant, while it may not be clear why all these "clouds" should be oriented with this specific geometry. Another possibility may involve a turbulent magnetic field. The component parallel to the shock front will be compressed not only in the transition region but also further in the recombination zone as the gas cools. The field component perpendicular to the shock front will not be affected and thus, there will be substantial variations of the magnetic pressure within the recombination zone. These variations will affect inversely the gas density distribution and consequently, the optical line emissivity will display a clumpy and irregular appearance (Raymond \& Curiel 1995).

The optical spectra showing strong [S II], [N II] and $\mathrm{H} \alpha$ emission and the calibrated optical images suggest that radiative shocks are present in $\mathrm{W} 44$. The neutral oxygen line at $6300 \AA$ is detected although it is difficult to establish accurately the actual source flux because the night sky is very bright in this line. In an attempt to determine the physical conditions giving rise to the optical emission we would have to explore the family of models appropriate for our case (e.g. Cox \& Raymond 1985; Raymond et al. 1988). Unfortunately, UV spectra and/or the [O III] $5007 \AA$ and [O II] $3727 \AA$ lines are not available and the actual family of radiative shocks cannot be confidently established. The observed spectra are characterized by the strong [N II] $6584 \AA$, [S II] 6716, $6731 \AA$ line emission relative to $\mathrm{H} \alpha$, the large sulfur line ratios implying low electron densities and the presence of moderate [O I] $6300 \AA$ emission. The latter line is marginally present in $100 \mathrm{~km} \mathrm{~s}^{-1}$ shocks, while in shocks faster than $\sim 150 \mathrm{~km} \mathrm{~s}^{-1}$ less flux in the $[\mathrm{NII}],[\mathrm{OI}]$ and $[\mathrm{S}$ II] lines is produced. Thus, we estimate shock velocities in the range of $110-150 \mathrm{~km} \mathrm{~s}^{-1}$ (Cox \& Raymond 1985). The ionization state of the preshocked 

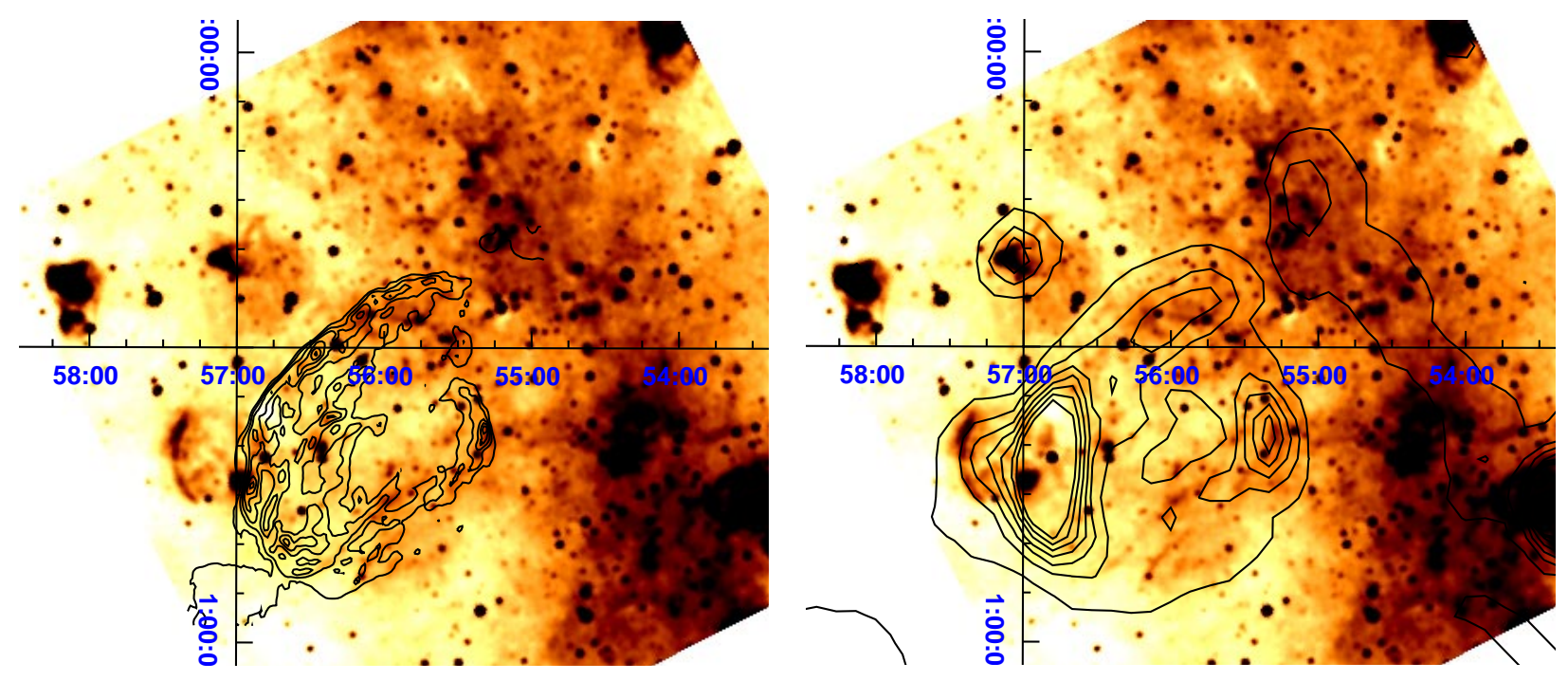

Fig. 4. The MSX data at $8 \mu \mathrm{m}$ are shown in this figure together with contours of radio emission at $1442 \mathrm{MHz}$ (15" resolution; left) and $4850 \mathrm{MHz}$ (3.5 resolution; right). The $1442 \mathrm{MHz}$ contours scale from 0.08 to $0.26 \mathrm{Jy} /$ beam, while the $4850 \mathrm{MHz}$ contours scale from 0.08 to $2.0 \mathrm{Jy} / \mathrm{beam}$.

gas cannot be resolved with the currrent data. The strong sulfur emission and the low electron densities may suggest the presence of moderate magnetic fields. Weak magnetic fields $(\sim 0.1-1 \mu \mathrm{G})$ allow for large compression factors in the recombination zone, while magnetic fields higher than $\sim 5 \mu \mathrm{G}$ suppress the compression resulting in lower electron and particle densities (e.g. Cox 1972; Raymond 1979; Raymond et al. 1988). Adopting electron densities around $200 \mathrm{~cm}^{-3}$ and the estimated shock velocities, we find preshock cloud densities of the order of 3 atoms per $\mathrm{cm}^{3}$ using the relation given by Fesen \& Kirshner (1980). However, this relation assumes negligible magnetic field and since it is probable that moderate magnetic fields are present what is obtained is actually a lower limit to the preshock could density. Shelton et al. (1999) estimate the velocity of the primary shock along the tenuous end to be $\sim 300 \mathrm{~km} \mathrm{~s}^{-1}$. Assuming pressure equilibrium between the ISM and the interstellar clouds, a density contrast of $\sim 4-7$ or greater is calculated.

Thermal emission from dust grains attributed to G 34.7-0.4 has been detected in the infrared by the IRAS satellite (Arendt 1989; Giacani et al. 1997). The dust grains can be collisionally heated by the primary blast wave which then emit in the infrared. Emission was present in all four IRAS bands but the moderate resolution of $\sim 2^{\prime}$ did not allow a detailed comparison of the optical and/or radio data with the dust emission. However, the Midcourse Space Experiment $\left({ }^{1} \mathrm{MSX}\right)$ provided data of higher sensitivity and spatial resolution $\left(\sim 20^{\prime \prime}\right)$ than the IRAS data. In Fig. 3 we show the MSX image at $8.28 \mu \mathrm{m}$ with the optical emission in $\mathrm{H} \alpha+[\mathrm{N}$ II $]$ as the overlaid contours. Faint filamentary structures appear to correlate well with the optical emission. In fact, there is an arc-like structure in the infrared which is partially correlated with the optical and radio emission of the remnant (Figs. 3 and 4). The arc originates from $\alpha \simeq 18^{\mathrm{h}} 55^{\mathrm{m}} 33^{\mathrm{s}}$, and $\delta \simeq 01^{\circ} 15^{\prime} 20^{\prime \prime}$, runs to the north for $\sim 18^{\prime}$ and then turns to the east up to $\alpha \simeq 18^{\mathrm{h}} 56^{\mathrm{m}} 13^{\mathrm{s}}$

\footnotetext{
1 The MSX data were retrieved from the NASA/IPAC infrared science archive at http://irsa.ipac.caltech.edu
}

and $\delta \simeq 01^{\circ} 32^{\prime} 33^{\prime \prime}$. The approximate radius of curvature of this arc is $\sim 11^{\prime}$. In addition, filamentary structures in the south also seem to match the radio emission at $1442 \mathrm{MHz}$ (Fig. 4). These structures are a few arcminutes long and $\sim 0$ '. 5 wide. If these structures are actually correlated to $\mathrm{G}$ 34.7-0.4 then temperatures around $10^{6} \mathrm{~K}$ would also be expected at the outer areas of the remnant. Another very interesting feature is located further to the east at $\alpha \simeq 18^{\mathrm{h}} 57^{\mathrm{m}} 25^{\mathrm{s}}$ and $\delta \simeq 01^{\circ} 18^{\prime} 15^{\prime \prime}$ extending for $\sim 10^{\prime}$ in the south-north direction. Even though, the high resolution $1442 \mathrm{MHz}$ data extend only up to $18^{\mathrm{h}} 57^{\mathrm{m}} 20^{\mathrm{s}}$, the lower resolution $4850 \mathrm{MHz}$ data match very well the position and curvature of this infrared filamentary structure. However, the nature of this structure (thermal or non-thermal) and its association to $\mathrm{G}$ 34.7-0.4 cannot be reliably determined using the currently available data. Dedicated radio spectral observations are needed to determine whether the extent of the remnant is larger in the east-west direction than we currently assume or the observed emission is simply projected on G 34.7-0.4. The irregular boundary of the radio emission in the west, northwest may be indicative of an interaction with the clouds in this area (Fig. 4, and also Seta et al. 1998). The interaction with molecular clouds in the east may be less extended (e.g. Wootten 1977) explaining the filamentary radio emission in this area. However, the possibility of interaction in the west is an open issue (e.g. Cox et al. 1999) and more kinematic data would be needed. For example, high resolution echelle spectra around the remnant's boundary would help to validate or discard this hypothesis by establishing the range of velocities in the east and the west.

\section{Conclusions}

Weak and diffuse emission is the major characteristic of the field of the supernova remnant G 34.7-0.4. The long-slit spectra verify the shock heated nature of the detected radiation and suggest low electron densities. The velocities of the shocks travelling into the interstellar clouds are in the range 
of $110-150 \mathrm{~km} \mathrm{~s}^{-1}$. The morphology of the images and the low electron densities implied by the spectra may indicate the presence of moderate magnetic fields. High resolution infrared data are rather well correlated with the optical and radio emission from G 34.7-0.4.

Acknowledgements. The authors would like to thank the referee for his comments and suggestions and E. B. Giacani and J. J. Condon for providing the $1442 \mathrm{MHz}$ and $4850 \mathrm{MHz}$ digital data, respectively. Skinakas Observatory is a collaborative project of the University of Crete, the Foundation for Research and Technology-Hellas and the Max-Planck-Institut für Extraterrestrische Physik. This research made use of data products from the Midcourse Space Experiment. Processing of the data was funded by the Ballistic Missile Defense Organization with additional support from NASA Office of Space Science. This research has also made use of the NASA/ IPAC Infrared Science Archive, which is operated by the Jet Propulsion Laboratory, California Institute of Technology, under contract with the National Aeronautics and Space Administration.

\section{References}

Arendt, R. G. 1989, ApJS, 70, 181

Condon, J. J., Broderick, J. J., Seielstad, G. A., Douglas, K., \& Gregory, P. C. 1994, AJ, 107, 1829

Cox, D. P., Shelton, R. L., Maciejewski, W., et al. 1999, ApJ, 524, 179

Cox, D. P., \& Raymond, J. C. 1985, ApJ, 298, 651

Cox, D. P. 1972, ApJ, 178, 143

Dickey, J. M., \& Lockman, F. J. 1990, ARAA, 28, 215

Fesen, R. A., \& Kirshner, R. P. 1980, ApJ, 242, 1023

Giacani, E. B., Dubner, G. M., Kassim, N. E., et al. 1997, AJ, 113, 1379
Hamuy, M., Walker, A. R., Suntzeff, N. B., et al. 1992, PASP, 104, 533

Hamuy, M., Suntzeff, N. B., Heathcote, S. R., et al. 1994, PASP, 106, 566

Harrus, I. M., Hughes, J. P., \& Helfand, D. J. 1996, ApJ, 464, L161

Harrus, I. M., Hughes, J. P., Singh, K. P., Koyama, K., \& Asaoka, I. 1997, ApJ, 488, 781

Jacoby, G. H., Quigley, R. J., \& Africano, J. L. 1987, PASP, 99, 672

Jones, L. R., Smith, A., \& Angellini, L. 1993, MNRAS, 265, 631

Kaler, J. B. 1976, ApJS, 31, 517

Koo, B.-C., \& Heiles, C. 1995, ApJ, 442, 679

Koo, B.-C., \& Heiles, C. 1991, ApJ, 382, 204

Kundu, M. R., \& Velusamy, T. 1972, A\&A, 20, 237

Lasker, B. M., Russel, J. N., \& Jenkner, H. 1999, in the HST Guide Star Catalog, version 1.1-ACT, The Association of Universities for Research in Astronomy, Inc.

Lynds, B. T. 1962, ApJS, 7, 1

Mavromatakis, F., Boumis, P., Papamastorakis, P., \& Ventura, J. 2002, A\&A, 388, 355

Petre, R., Kuntz, K. D., \& Shelton, R. L. 2002, ApJ, 579, 404

Predehl, P., \& Schmitt, J. H. M. M. 1995, A\&A, 293, 889

Raymond, J. C., Hester, J. J., Cox, D., et al. 1988, ApJ, 324, 869

Raymond, J. C. 1979, ApJS, 39, 1

Raymond, J. C., \& Curiel, S. 1995, The Fifth Mexico-Texas Conference on Astrophysics: Gaseous Nebulae and Star Formation, 3, 199

Rho, J., Petre, R., Schlegel, E. M., \& Hester, J. J. 1994, ApJ, 430, 757

Seta, M., Hasegawa, T., Dame, T. M., et al. 1998, ApJ, 505, 286

Shelton, R. L., Cox, D. P., Maciejewski, W., et al. 1999, ApJ, 524, 192

Tsunemi, H., \& Enoguchi, H., Cospar 2002, Houston, Texas

Wolszczan, A., Cordes, J. M., \& Dewey, R. J. 1991, ApJ, 372, L99

Wootten, H. A. 1977, ApJ, 216, 440 